\title{
Aislamiento e Identificación Bioquímica de Cepas de Pasteurella multocida y Gallibacterium anatis en Aves de Producción con Signos Respiratorios
}

\author{
Isolation ANd Biochemical ChaRaCterization of PASTEURELla MULTOCIDA AND \\ Gallibacterium anatis Strains in Poultry with Respiratory Signs
}

\author{
Gonzalo Castillo $^{1,3}$, Ysabel Koga ${ }^{1,4}$, Arnaldo Alvarado ${ }^{1,2}$, Robert Tinoco ${ }^{1}$, \\ Daniel Fernández ${ }^{1}$
}

\section{Resumen}

\begin{abstract}
El objetivo del estudio fue determinar las biovariedades de Pasteurella multocida y Gallibacterium anatis en aves de producción con signos respiratorios. Estas bacterias fueron aisladas de muestras de secreciones y órganos de pollos de carne, gallinas de postura y patos criollos afectados, provenientes de granjas avícolas de la costa y selva del Perú. De 25 aislamientos se identificaron 13 cepas de P. multocida y 12 de G. anatis, mediante características de cultivo, morfología y pruebas bioquímicas (oxidasa, catalasa, indol, ureasa). Las cepas fueron sometidas a la prueba de fermentación en micropozos, consistente en una batería de 10 carbohidratos y un aminoácido para su tipificación. Los resultados indican que ocho cepas de $P$. multocida correspondieron a la biovariedad $1 \mathrm{y}$ las restantes a las biovariedades 3, 4, 6, 10 y 11, en tanto que 11 cepas de G. anatis correspondieron a la biovariedad haemolytica y la otra a la biovariedad anatis.
\end{abstract}

Palabras clave: pollos de carne, gallinas, patos, fermentación microbiana, síndrome respiratorio aviar

\section{Abstract}

The aim of the study was to identify biovars of Pasteurella multocida and Gallibacterium anatis in poultry with respiratory signs. These bacteria were isolated from samples of secretions and organs of affected broilers, layers and ducks from various poultry farms in the coast and the tropics of Peru. Out of 25 isolates obtained, 13 strains

\footnotetext{
${ }^{1}$ Bioservice SRL, Lima

${ }^{2}$ Laboratorio de Patología Clínica y Biología Molecular, Facultad de Medicina Veterinaria, Universidad Nacional Mayor de San Marcos, Lima, Perú

${ }^{3}$ Carrera Profesional de Medicina Veterinaria, Facultad de Ciencias Agropecuarias, Universidad Alas Peruanas, Lima, Perú

${ }^{4}$ E-mail: ysabelkogay@gmail.com
}

Recibido: 10 de diciembre de 2013

Aceptado para publicación: 18 de junio de 2014 
of P. multocida and 12 of G. anatis were identified based on culture characteristics, morphology and biochemical tests (oxidase, catalase, indole, urease). These strains were subjected to micro-well fermentation tests with 10 carbohydrates and one amino acid for typification. Results showed that eight strains of $P$. multocida corresponded to biovar 1 and the others to biovars 3, 4, 6, 10 and 11, while 11 strains of $G$. anatis corresponded to biovar haemolytica and one strain to biovar anatis.

Key words: broiler, hen, duck, microbial fermentation, avian respiratory syndrome

\section{INTRODUCCIÓN}

La mejora genética para obtener aves de alta producción y las condiciones de manejo pueden predisponer a la aparición del síndrome respiratorio (SRA), el cual puede ser producido por una combinación de infecciones virales (Newcastle, Bronquitis infecciosa, Rinotraqueitis aviar, y Laringotraqueitis, entre otras) y bacterianas (Avibacterium, Pasteurella, Ornithobacterium rhinotracheale, Mycoplasma y Bordetella, entre otras) (Muhairwa et al., 2002). Asimismo, en forma individual, estos microorganismos pueden causar signos y lesiones clínicas similares (Muhairwa et al., 2002).

Las especies Pasteurella multocida y Gallibacterium anatis tienen una participación importante en el SRA y son aislados con frecuencia en el análisis microbiológico. Estos microorganismos causan entre 15 y $20 \%$ de mortalidad. Las aves que sobreviven ocasionan una disminución en el comportamiento reproductivo afectando la productividad de las granjas (Vásquez et al., 2001; Bojesen et al., 2003; Bisgaard et al., 2005), además de permanecer como portadoras (Calnek, 2000).

P. multocida es el agente infeccioso del cólera aviar, presente mayormente en pollos de carne y pavos (Blackall y Mifflin, 2000; Jonas et al., 2001; Shivachandra et al., 2005; Corney et al., 2007). Esta bacteria fue divi- dida en tres subspecies (multocida, septica y gallicida) en 1985, y se diferencian por su habilidad de fermentar carbohidratos (Vadillo et al., 2002; Quinn et al., 2005; Ekundayo et al., 2008); además, se reconocen 15 biovares de estas subespecies, cuya denominación va del 1 al 15 .

G. anatis ha sido reportada como un organismo aviar semejante a $P$. haemolytica, A. salpingitidis o $P$. anatis, pero actualmente se encuentra incluida dentro del nuevo género Gallibacterium de la familia Pasteurellaceae (Christensen et al., 2003). Este género solo contiene la especie $G$. anatis con dos biovares (Christensen et al., 2003), que se diferencian por fermentar determinados sustratos y por su acción hemolítica positiva ( $G$. anatis biovar haemolytica) o negativa ( $G$. anatis biovar anatis) (Bisgaard et al., 2005; García-Gómez et al., 2005; Bojesen et al., 2007; Rzewuska et al., 2007).

Dado que las bacterias involucradas en el SRA no producen signos y lesiones clínicas patognomónicas, es casi imposible realizar un efectivo diagnóstico clínico, de allí que se necesita identificar los patógenos a través de pruebas de laboratorio (Calnek, 2000). Asimismo, es importante determinar su presencia y frecuencia de los biovares en aves en el país. El objetivo de esta investigación fue determinar los biovares de $P$. multocida y $G$. anatis presentes en las aves comerciales de la costa y de la selva del Perú. 


\section{Materiales y Métodos}

Se analizaron 25 casos respiratorios en pollos de carne, gallinas ponedoras y patos de granjas localizadas en los departamentos peruanos de Lima (17 casos), La Libertad (5), Ica (1), Tacna (1) y Ucayali (1), en un lapso de cinco meses. El tamaño de las empresas en términos de número de aves era muy diverso. En cada caso, se tomaron hisopados de barbillas, senos infraorbitales, tráqueas, pulmones y sacos aéreos, así como de improntas de hígado y bazo de las aves afectadas.

Las muestras se trabajaron en los Laboratorios Bioservice, Lima. Fueron cultivadas en agar sangre y agar MacConkey e incubadas en condiciones anaeróbicas a $37{ }^{\circ} \mathrm{C}$ por 24 a 48 h (Carter y Cole, 1990; Diallo y Frost, 2000). Se hizo el análisis de las características microscópicas de las colonias (Quentin y Russel, 1991), seguido del análisis bioquímico de oxidasa, catalasa, indol y ureasa (Vadillo et al., 2002).

Las cepas aisladas fueron sometidas a pruebas de fermentación de carbohidratos: glucosa, arabinosa, dulcitol, lactosa, maltosa, manitol, sorbitol, sacarosa, trehalosa y xilosa, y el aminoácido ornitina (Calnek, 2000). La capacidad de fermentación de las cepas se midió a través de un medio basal consistente en peptona al $1 \%, \mathrm{NaCl}$ al $0.5 \%$, púrpura de bromocresol al $1 \%$ y carbohidrato/aminoácido al $20 \%$ con $\mathrm{pH}$ de 6.8. En un microtubo por cada sustrato se dispensó $200 \mu 1$ de medio basal, $10 \mu 1$ del carbohidrato o aminoácido y $10 \mu 1$ del inóculo (una colonia de cada cepa se diluyó en $2 \mathrm{ml}$ de caldo nutritivo). Se incubaron a $37{ }^{\circ} \mathrm{C}$ por 24 a $48 \mathrm{~h}$. Un color amarillo se consideró como reacción positiva y un color púrpura como reacción negativa.

\section{Resultados}

Se aislaron e identificaron 25 cepas de P. multocida y G. anatis. De estas, 13 fue ron identificadas como biovar 1 (8 aislamientos), 3, 4, 6, 10 y 11 (un aislamiento por biovar) de $P$. multocida, mientras que 12 cepas correspondieron a $G$. anatis biovar haemolytica (11 aislamientos) y anatis (1 aislamiento). En ningún caso ocurrió una infección mixta. El Cuadro 1 muestra la procedencia por departamento de los aislamientos de $P$. multocida y de G. anatis.

Las cepas de $P$. multocida se distinguieron por su habilidad de fermentar arabinosa, maltosa, sorbitol, trehalosa y xilosa, lo que permitió la identificación de seis biovares (Cuadro 2), donde seis de ellos fueron de la

Cuadro 1. Procedencia de aislamientos de Pasteurella multocida y de Gallibacterium anatis de aves afectadas con procesos respiratorios en cinco departamentos del Perú

\begin{tabular}{lcc}
\hline Departamento & P. multocida & G. anatis \\
\hline Ica & 1 & 0 \\
La Libertad & 3 & 2 \\
Lima & 7 & 10 \\
Tacna & 1 & 0 \\
Ucayali & 1 & 0 \\
\hline
\end{tabular}

subespecie multocida, no pudiéndose determinar la subespecie de los biovares 6 y 11 . Por otro lado, las cepas de $G$. anatis se diferenciaron por los resultados en la fermentación de lactosa, maltosa y trehalosa (Cuadro 3).

\section{Discusión}

Once de las 13 cepas de $P$. multocida correspondieron a la subespecie multocida, lo que sugiere que podría ser la subespecie más frecuente en patologías respiratorias aviares en el país; sin embargo, se debe tener en cuenta el reducido número de mues- 
Cuadro 2. Identificación de biovares ${ }^{1}$ de Pasteurella multocida en aislados de muestras de aves afectadas con procesos respiratorios en cinco departamentos del Perú

\begin{tabular}{llllllll}
\hline & & \multicolumn{7}{c}{ Biovar $^{2}$} \\
\cline { 3 - 8 } Acidificación & Glucosa & + & 3 & 4 & 6 & 10 & 11 \\
& Arabinosa & - & - & - & - & - & + \\
& Dulcitol & - & - & - & - & - & - \\
& Lactosa & - & - & - & - & - & - \\
& Maltosa & - & - & - & - & + & - \\
& Manitol & + & + & + & + & + & + \\
& Sorbitol & + & + & + & - & - & + \\
& Sacarosa & + & + & + & + & + & + \\
& Trehalosa & - & - & + & - & + & - \\
& Xilosa & - & + & - & - & + & - \\
ODC $^{3}$ & & + & + & + & + & + & + \\
\hline
\end{tabular}

${ }_{1}^{1}$ Fegan et al. (1995)

${ }^{2}$ Resultados en base a 8 aislamientos del biovar 1 y de un aislamiento para cada uno de los otros biovares

${ }^{3}$ Ornitine decarboxilation

Cuadro 3. Identificación de biovares ${ }^{1}$ de Gallibacterium anatis en aislados de muestras de aves afectadas con procesos respiratorios en cinco departamentos del Perú

\begin{tabular}{ccc}
\hline & \multicolumn{2}{c}{ Biovar } \\
\cline { 2 - 3 } & $\begin{array}{c}\text { Haemolytica } \\
(\mathrm{n}=11 \text { cepas })\end{array}$ & $\begin{array}{c}\text { Anatis } \\
(\mathrm{n}=1 \text { cepa })\end{array}$ \\
\hline Acidificación & & + \\
Glucosa & + & - \\
Arabinosa & - & - \\
Lactosa & + & - \\
Maltosa & + & + \\
Manitol & + & + \\
Sacarosa & + & - \\
Trehalosa & + & + \\
Xilosa & + & - \\
ODC $^{2}$ & - & \\
\hline
\end{tabular}

${ }^{1}$ Blackall et al. (2008)

${ }^{2}$ Ornitine decarboxilation 
tras. No obstante, esta subespecie ha sido reportada como la más frecuente en Australia (Blackall et al., 1995; Fegan et al., 1995), Estados Unidos (Aye et al., 2001), Indonesia (Jonas et al., 2001) y Nigeria (Ekundayo et al., 2008). Ninguna de las cepas evaluadas en el presente estudio correspondió a las subespecies septica y gallicida, las cuales se hallaron en los estudios mencionados de Indonesia y Nigeria.

Se ha descrito que la subespecie multocida involucra los biovares 1, 2, 3, 4, 9, $10,12,13$ y 14 , la subespecie septica contiene el biovar 7 y la subespecie gallicida el biovar 8 (Fegan et al., 1995). De los seis biovares identificados, el biovar 1 fue el más común, resultado que concuerda con los hallazgos de Jonas et al. (2001), donde este biovar se encontró en 6 de las 9 cepas evaluadas de $P$. multocida; sin embargo, en el trabajo de Fegan et al. (1995), los biovares 2 y 3 fueron los más comunes, produciendo lesiones más severas en los órganos comprometidos. Por otro lado, los biovares 3, 4, 6, 10 y 11, encontrados en el presente estudio, fueron asimismo identificados por Fegan et al. (1995), con la excepción del biovar 11. El biovar 1 fue aislado de diversos órganos, lo que confirma su potencial acción septicémica.

Con respecto a las 12 cepas de $G$. anatis, el biovar haemolytica predominó con 11 aislamientos sobre uno del biovar anatis. Esta predominancia también fue observada por Rzewuska et al. (2007), donde aislaron 13 cepas de G. anatis en pavos reales con síntomas respiratorios, y todas ellas fueron fenotípicamente identificadas como biovar haemolytica. En todo caso, se podría argumentar que los pavos son menos susceptibles a las infecciones respiratorias causadas por $G$. anatis que las aves de corral y que pueden actuar como una fuente de transmisión de este microorganismo. Por otro lado, la severidad de las lesiones y los diversos órganos donde se aisló la bacteria, sugieren la capacidad invasiva de G. anatis, principalmente en el caso del biovar haemolytica (Bojesen et al., 2004; Bojesen y Shivaprasad, 2007).

\section{Conclusiones}

- Se identificaron 13 cepas de $P$. multocida y 12 cepas de G. anatis.

- Ocho cepas de P. multocida correspondieron al biovar 1 y las otras a los biovares 3, 4, 6, 10 y 11 .

- De 12 cepas de G. anatis, 11 fueron identificadas como biovar haemolytica y solo una correspondió al biovar anatis.

\section{Agradecimiento}

Los autores agradecen al Dr. Patrick J. Blackall por facilitar los cuadros de identificación bioquímica de las subespecies de $P$. multocida y G. anatis.

\section{Literatura Citada}

1. Aye PE, Angrick T, Morishita T, Harr B. 2001. Prevalence and characteristics of Pasteurella multocida in commercial turkeys. Avian Dis 45: 182-190.

2. Bisgaard M, Christensen H, Bojesen AM, Christensen JP. 2005. Avian infections by species of Pasteurellaceae, an update. Avian Dis 49: 534-539.

3. Blackall PJ, Pahoff JL, Marks D, Fegan N, Morrow CJ. 1995. Characterization of Pasteurella multocida isolated from fowl cholera outbreaks on turkey farms. Austr Vet J 72: 135-138.

4. Blackall P, Mifflin J. 2000. Identification and typing of Pasteurella multocida: a review. Avian Pathol 29: 271-287.

5. Blackall PJ, Norskow-Lauritsen N. 2008. Pasteurellaceae - the view from the diagnostic laboratory. En: Kuhnert P, 
Christensen H (eds). Pasteurellaceae: biology, genomics and molecular aspects. Norwich, UK: Horizon Scientific Press. p 227-259.

6. Bojesen AM, Nielsen SS, Bisgaard M. 2003. Prevalence and transmission of haemolytic Gallibacterium species in chicken production systems with different biosecurity levels. Avian Pathol 32: 503-510.

7. Bojesen AM, Nielsen O, Christensen J, Bisgaard M. 2004. In vivo studies of Gallibacterium anatis. Avian Pathol 33: 145-152.

8. Bojesen A, Shivaprasad H. 2007. Genetic diversity of Gallibacterium isolates from California turkeys. Avian Pathol 36: 227-230.

9. Bojesen AM, Vásquez ME, Robles $F$, Gonzales C, Soriano EV, Olsen JE, Christensen $H$. 2007. Specific identification of Gallibacterium by PCR using primers targeting the 16S rRNA genes. Vet Microbiol 123: 262-268.

10. Calnek B. 2000. Enfermedades de las aves. $2^{\text {a }}$ ed. México: Ed Manual Moderno. $1067 \mathrm{p}$.

11. Carter G, Cole J. 1990. Diagnostic procedures in veterinary bacteriology and mycology. $5^{\text {th }}$ ed. San Diego, USA: Academic Press Ltd. 305 p.

12. Christensen H, Bisgaard M, Bojesen A, Mutters R, Elmerdahl J. 2003. Genetic relationships among avian isolates classified as Pasteurella haemolytica, «Actinobacillus salpingitidis» or Pasteurella anatis with proposal of Gallibacterium anatis gen. nov., comb. nov. and description of additional genomospecies within Gallibacterium gen. nov. Int J Syst Evol Microbiol 53: 275-287.

13. Corney BG, Diallo IS, Wright LL, Hewitson GR, De Jong AR, Burrell PC, Duffy PF, et al. 2007. Pasteurella multocida detection by 5' Taq nuclease assay: a new tool for use in diagnosing fowl cholera. J Microbiol Methods 69: 376-380.
14. Diallo I, Frost A. 2000. Characteristics of a haemolytic extract from avian Pasteurella multocida. Vet Microbiol 72: 37-45.

15. Ekundayo S, Odugbo M, Olabode A, Okewole P. 2008. Phenotypic variability among strains of Pasteurella multocida isolated from avian, bovine, caprine, leporine and ovine origin. Afr $\mathrm{J}$ Biotechnol 7: 1347-1350.

16. Fegan N, Blackall PJ, Pahoff Jl. 1995. Phenotypic characterisation of Pasteurella multocida isolates from Australian poultry. Vet Microbiol 47: 281-286.

17. García-Gómez E, Vaca S, PérezMéndez A, Ibarra-Caballero J, PérezMárquez V, Tenorio V, NegreteAbascal E. 2005. Gallibacterium anatis-secreted metalloproteases degrade IgG. Avian Pathol 34: 426-429.

18. Jonas M, Morishita T, Angrick W, Jahja J. 2001. Characterization of nine Pasteurella multocida isolates from avian cholera outbreaks in Indonesia. Avian Dis 45: 34-42.

19. Muhairwa A, Christensen JP, Bisgaard M. 2002. Serum resistence of Pasteurella multocida in avian and porcine sera, and comparative virulence investigations of selected serumsensitive and resistant strains in chickens. Avian Pathol 31: 183-191.

20. Quentin N, Russel M, 1991. Bacteriología y micología médicas. $2^{\text {da }}$ ed. México DF: McGraw-Hill Interamericana. $713 \mathrm{p}$.

21. Quinn P, Markey B, Carter M, Donelly W, Leonard F. 2005. Microbiología y enfermedades infecciosas veterinarias. Zaragoza: Acribia. $667 \mathrm{p}$.

22. Rzewuska M, Karpinska E, Szeleszczuk P, Binek M. 2007. Isolation of Gallibacterium spp from peacocks with respiratory tract infections. Medycyna Wet 63: 1431-1433.

23. Shivachandra SB, Kumar AA, Gautam R, Saxena MK, Chaudhuri P, Srivastava SK. 2005. Detection of 
multiple strains of Pasteurella multocida in fowl cholera outbreaks by polymerase chain reaction-based typing. Avian Pathol 34: 456-462.

24. Vadillo S, Píriz S, Mateos E. 2002. Manual de microbiología veterinaria. Madrid: McGraw-Hill Interamericana. 843 p.
25. Vásquez M, Campogarrido $M$, Cubillas C, Coss J, Gonzales C, Sivanandan V. 2001. Reporte de un caso de Pasteurella haemolytica en gallina de postura (Babcock) en Tehúacan Puebla. ANECA Symposium, México DF. 